\title{
A utilização do álcool como mediador social entre universitários
}

\author{
Wellington Danilo Soares ${ }^{1}$ \\ Carolina Júnia Reis Paz ${ }^{1}$ \\ Ludmila Cotrim Fagundes ${ }^{1}$ \\ Daniel Antunes Freitas ${ }^{1}$ \\ Kimberly Marie Jones ${ }^{1}$ \\ Henrique Andrade Barbosa ${ }^{1}$
}

Introdução: o consumo de álcool entre adolescentes é maior entre os universitários e gera consequências acadêmicas negativas. Os hábitos de consumo adotados nesse período da vida são decisivos, já que, em sua maioria, são mantidos na vida adulta. Objetivo: avaliar a produção científica acerca da utilização do álcool como mediador social entre universitários. Método: revisão integrativa realizada nas bases de dados Literatura Latino-Americana e do Caribe em Ciências da Saúde (LILACS), PUBMED e Scientific Eletronic Library Online (SciELO), com duas combinações dos cinco descritores: Consumo de bebidas alcoólicas; AND Estudantes; AND Educação Superior; AND Socialização; AND Álcool. Resultados: na busca inicial, encontraram-se 665 artigos; após análise criteriosa e aplicação dos critérios de seleção, 21 foram utilizados. Como delineamento, prevaleceram 14 estudos qualitativos, 5 ensaios controlados randomizados, 1 não randomizado e 1 coorte. Conclusão: o alcoolismo entre universitários constitui um problema no âmbito da educação e saúde e que precisa de ações efetivas a fim de controlar suas consequências em curto e longo prazo.

Descritores: Estudantes; Socialização; Álcool.

\footnotetext{
${ }^{1}$ Universidade Estadual de Montes Claros, Montes Claros, MG, Brasil.
} 


\section{The use of alcohol as a social mediator among university students}

Introduction: alcohol consumption is higher among college students and generates negative consequences. The habits adopted during this period of life are decisive because, for the most part, they will be maintained throughout life. Objective: to evaluate the scientific production about the use of alcohol as social mediator among college students. Method: integrative review made in the Latin American and Caribbean Health Sciences Literature (LILACS), PUBMED and Scientific Eletronic Library Online (SciELO). Two combinations of the five descriptors were used, namely: Alcohol consumption; AND Students; AND Higher Education; AND Socialization; AND Alcohol. Results: a total of 665 articles were found in the initial search; after careful analysis and application of the selection criteria, 21 articles were used. As for the design, there were 14 qualitative studies, 5 randomized controlled trials, 1 non-randomized trial and 1 cohort study, prevailed. Conclusion: alcoholism among college students is a problem in education and health and requires effective action to control its short- and long-term consequences.

Descriptors: Students; Socialization; Alcohol.

\section{El uso de alcohol como mediador social entre universitarios}

Introducción: el consumo de alcohol entre adolescentes es mayor entre los universitarios y genera consecuencias académicas negativas. Los hábitos de consumo adoptados en ese período de la vida son decisivos, ya que, en su mayoría, se mantienen en la vida adulta. Objetivo: evaluar la producción científica sobre el uso del alcohol como mediador social entre universitarios. Método: revisión integrativa realizada en las bases de datos Literatura Latino-Americana y de Caribe en Ciencias de la Salud (LILACS), PUBMED y Scientific Eletronic Library Online (SciELO). Con dos combinaciones de los cinco descriptores Consumo de bebidas alcohólicas; AND Estudiantes; AND Educación Superior; AND Socialización; AND Alcohol. Resultados: en la búsqueda inicial se encontraron 665 artículos; después de un análisis riguroso y de la aplicación de los criterios de selección, 21 se utilizaron. Como delineamiento, prevalecieron 14 estudios cualitativos, 5 ensayos controlados aleatorizados, 1 no randomizado y 1 cohorte. Conclusión: El alcoholismo entre universitarios constituye un problema en el ámbito de la educación y la salud y que necesita acciones efectivas para controlar sus consecuencias a corto y largo plazo.

Descriptors: Estudiantes; Socialización; Alcohol.

\section{Introdução}

O consumo de álcool entre adolescentes relacionase, negativamente, com desempenho acadêmico, integridade física e comportamentos sexuais( ${ }^{(1)}$. Estudos recentes apontam que o uso dessas substâncias são maiores nessa faixa etária, quando comparados com os adultos, e que, entre os adolescentes, as taxas são maiores para os acadêmicos ${ }^{(2)}$.

Outro fator de risco entre os adolescentes é a timidez, a qual caracteriza-se por problemas de internalização, ansiedade, depressão e autopercepção baixa. Apesar do consumo ser menos frequente entre esses indivíduos, os tímidos são mais propensos a 
beber em eventos sociais ${ }^{(3)}$ com esperanças positivas para a sociabilidade e a sexualidade ${ }^{(4)}$.

Como coadjuvante das consequências do abuso do álcool entre adolescentes, a tecnologia atual aumenta a suscetibilidade para se envolver em comportamentos sociais lamentáveis quando intoxicados. A exemplo, mensagens de texto, ligações ou postagens bêbadas que expõem o jovem a inconvenientes. Nesse contexto, o estímulo à recordação de situações constrangedores que vivenciaram quando embriagados pode ser uma maneira mais eficaz de conseguir um arrependimento antecipado do que alertas sobre consequências mais graves, já que o mais comum pode ter influência maior na modulação das ações dos adolescentes em campanhas de conscientização(4).

O melhor preditor para o consumo de álcool entre universitários parece ser o consumo antes da faculdade, juntamente com os variáveis problemas da fase de transição: impulsividade, busca de sensações, instabilidade no humor e ansiedade. Além dos jogos alcoólicos, que incentivam com ruídos, competição, integração social e emoção(5), e das redes sociais ${ }^{(6)}$.

Consequentemente, a morbidade e mortalidade aumentam entre universitários que bebem e a predisposição à permanência desse consumo na vida adulta é alta(7). Sobre esse aspecto, pode-se correlacionar uma Teoria das Representações Sociais de Serge Moscovici (1978) intitulada A Psicanálise, sua imagem e seu público. Nessa perspectiva, as representações sociais são reconhecidas como fenômenos psicossociais, históricos e culturalmente condicionados, o que pode definir a bebida alcoólica como um agente complexo que, ao mesmo tempo em que favorece os momentos inter-relacionais, determina preconceitos em relação a quem abusa da bebida e que culturalmente está ligada à masculinidade do homem, isso explica a maior mortalidade relacionada ao álcool nesse gênero ${ }^{(8)}$. Ademais, a hereditariedade demonstra ter influência no abuso dessa substância(9).
Para tanto, este estudo teve como objetivo avaliar a produção científica acerca da utilização do álcool como mediador social entre universitários, uma vez que se torna preocupante a utilização dessa substância entre o público citado.

\section{Métodos}

Realizou-se uma revisão integrativa da literatura por meio de pesquisa e avaliação criteriosa de estudos divulgados sobre a hipótese proposta. A avaliação dos resultados encontrados possibilita a execução das evidências abordadas na prática. A questão norteadora definida para a investigação do estudo foi "Utilização do Álcool como Mediador Social entre Universitários".

Para atingir a precisão metodológica do estudo, seguiram-se as seis etapas propostas, sendo (1) estabelecimento de hipótese ou questão de pesquisa; (2) amostragem ou busca na literatura; (3) categorização dos estudos; (4) avaliação dos estudos incluídos na revisão; (5) interpretação dos resultados; e (6) síntese do conhecimento ou apresentação da revisão. Nesse contexto, utilizaram-se as bases de dados virtuais: Literatura Latino-Americana e do Caribe em Ciências da Saúde (LILACS), PUBMED e Scientific Electronic Library Online (SciELO).

A busca por trabalhos nesta revisão literária foi orientada conforme a combinação de 05 (cinco) descritores, aplicando-se moduladores booleanos, indexados no DeCS (Descritores em Ciências da Saúde), utilizando-se linguagem única na indexação de artigos de revistas científicas, livros, anais de congressos, relatórios técnicos e outros tipos de materiais, assim como em pesquisas e recuperação de assuntos da literatura científica. Usaram-se duas chaves de busca no total, pesquisadas nos idiomas Inglês, Espanhol e Português. Para a realização do rastreio, os descritores foram utilizados conforme apresentados na Figura 1.

\begin{tabular}{|c|c|c|}
\hline DESCRITORES EM PORTUGUÊS & DESCRITORES EM INGLÊS & DESCRITORES EM ESPANHOL \\
\hline $\begin{array}{c}\text { Consumo de bebidas alcoólicas AND } \\
\text { Estudantes AND Educação Superior }\end{array}$ & $\begin{array}{c}\text { Alcohol Drinking AND Students } \\
\text { AND Education, Higher }\end{array}$ & $\begin{array}{c}\text { Consumo de bebidas alcoholicas AND } \\
\text { Estudiantes AND Educacion Superior }\end{array}$ \\
\hline $\begin{array}{c}\text { Socialização AND Álcool AND } \\
\text { Estudantes }\end{array}$ & $\begin{array}{c}\text { Socialization AND Alcohol AND } \\
\text { Students }\end{array}$ & $\begin{array}{c}\text { Socialización AND Álcool AND } \\
\text { Estudiantes }\end{array}$ \\
\hline
\end{tabular}

Figura 1 - Descritores utilizados na busca 


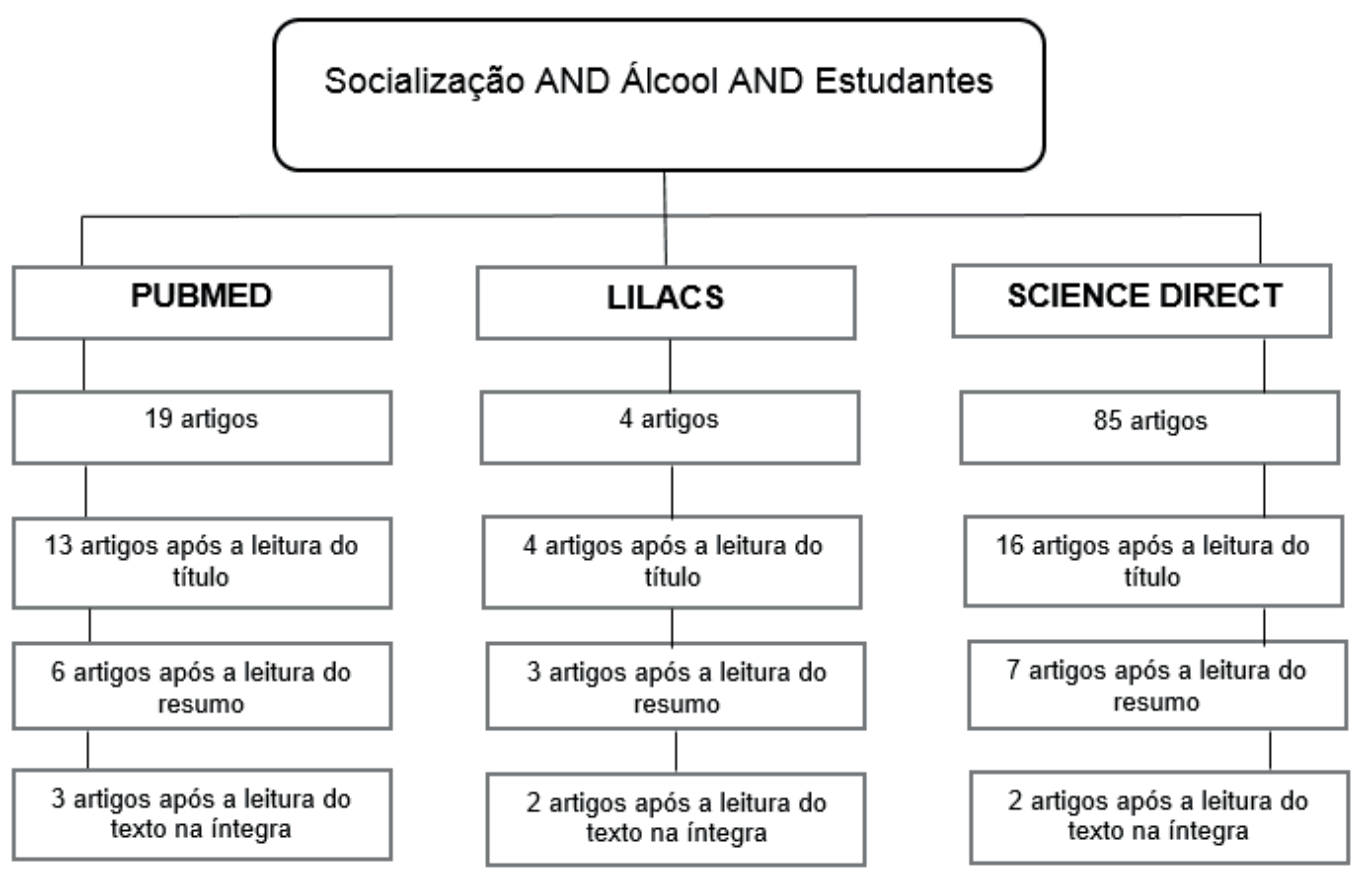

Figura 2 - Fluxograma sintetizando as estratégias de busca utilizadas com a combinação "Socialização AND Álcool AND Estudantes", que resultaram na amostra de dados final

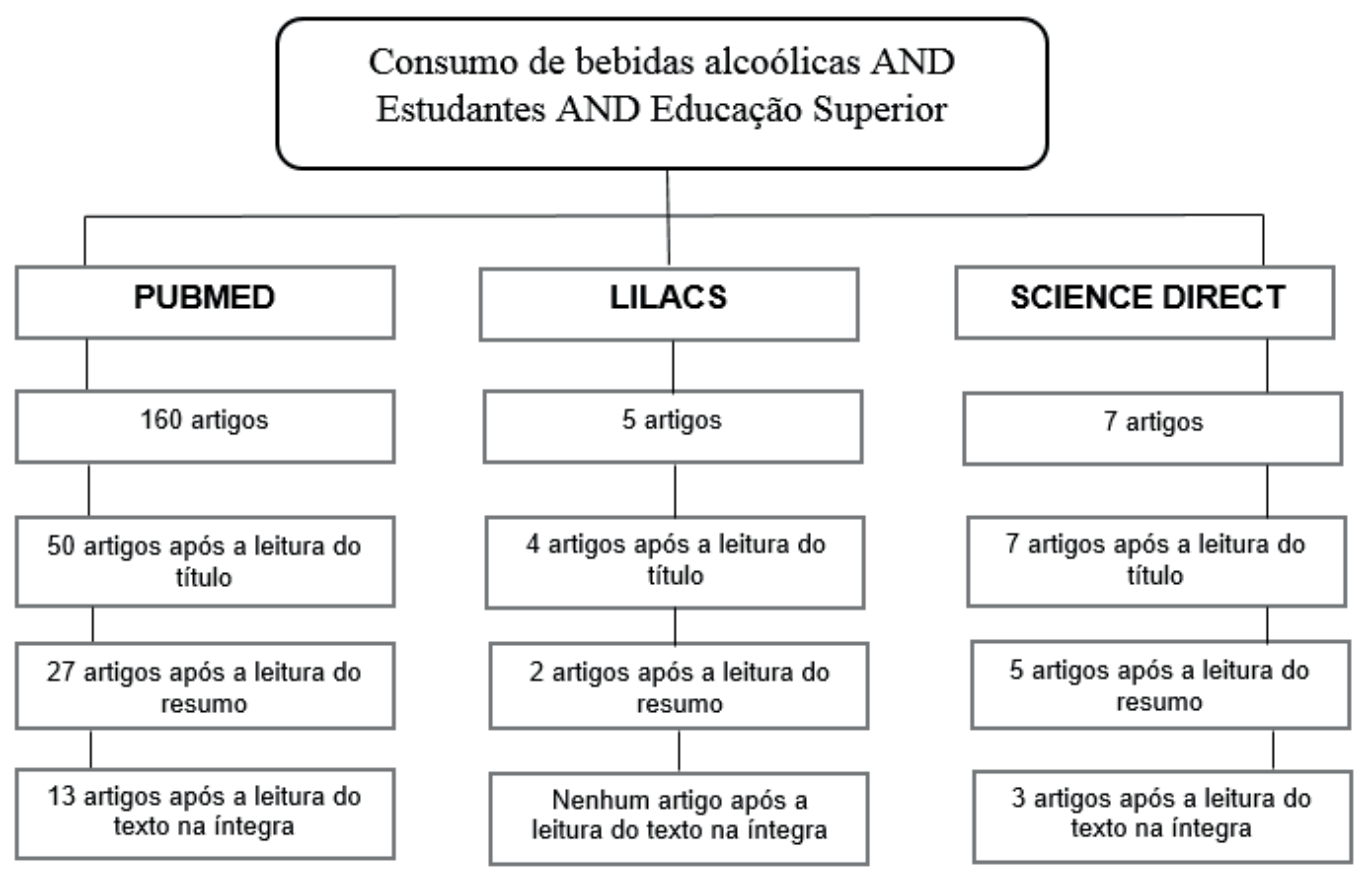

Figura 3 - Fluxograma sintetizando as estratégias de busca utilizadas com a combinação "Consumo de bebidas alcoólicas AND Estudantes AND Educação Superior“, que resultaram na amostra de dados final

A análise dos dados seguiu critérios de inclusão baseados no tema oposto pela presente pesquisa, sendo (1) estudos realizados entre os anos 2012 e 2017; (2) que possuíam texto completo disponível online; e (3) publicados nos idiomas Inglês, Português ou Espanhol. Excluíram-se os estudos de revisão sistemática da literatura, artigos repetidos e trabalhos que apenas tangenciavam o tema.
Após a análise dos trabalhos, aplicados os critérios de inclusão e exclusão, encontraram-se 665 artigos. A primeira etapa da triagem selecionou um total de 95 artigos com base na leitura dos títulos dos mesmos. Posteriormente, realizou-se a leitura dos resumos apresentados nos artigos selecionados, separando-se 50 trabalhos. Ao final do processo, foram escolhidos e analisados integralmente 23 artigos, e após a exclusão 
dos trabalhos repetidos, a amostra final contou com 21artigos.

Para análise qualitativa dos artigos da amostra final, fundamentou-se nos níveis de evidência propostos por Stillwel|(10): I - Revisão sistemática ou metanálise; II - Ensaio clínico randomizado controlado; III - Ensaio clínico controlado sem randomização; IV - Caso controle ou estudo de coorte; $\vee$ - Revisão sistemática de estudo qualitativo ou descritivo; VI - Estudo qualitativo ou descritivo; VII - Artigo de opinião ou consenso de órgãos governamentais ou conselho de especialidades médicas. Os dados obtidos após leitura dos artigos foram apresentados na forma de resumo descritivo em tabelas que incluem as informações do nível de evidência e ano do estudo (Figuras 2 e 3 ).

\section{Caracterização dos estudos}

A pesquisa com os descritores nas bases de dados resultou em um total de 665 artigos encontrados cuja temática se relacionava com uso de álcool entre estudantes universitários, suas causas e consequências. Desses artigos, $26,91 \%(n=179)$ foram encontrados na base de dados PUBMED, 1,36\% $(n=9)$ na base de dados LILACS/SCiELO e 71,73\% ( $n=477)$ na base de dados Science Direct. Para a seleção dos artigos, realizou-se a leitura, primeiramente, dos títulos, depois dos resumos e, por último, do corpo do texto na íntegra, excluindo artigos repetidos e que não se enquadravam com a temática. O processo de seleção gerou uma amostra final de $3,15 \%(n=21)$ do total de artigos encontrados; destes, $69,57 \%(n=16)$ constavam na base de dados PUBMED, 8,69\% ( $\mathrm{n}=2)$ na base de dados LILACS/ SciElO e $21,74 \%(n=5)$ na base de dados Science Direct. Dessa forma, somando-se os artigos encontrados nas bases de dados, obtém-se um total de 23 artigos encontrados. Após a correção dos 2 artigos repetidos, restou a amostra final de 21 artigos.

Com referência ao ano de publicação dos artigos selecionados dentro da amostra final, demonstrado na Tabela 1, observa-se que as publicações foram bem distribuídas entre o período em questão, não havendo nenhum ano sem publicação.

Tabela 1 - Distribuição dos artigos conforme o ano de publicação

\begin{tabular}{ccc}
\hline Ano & $\mathbf{n}$ & $\%$ \\
\hline 2012 & 2 & 9,53 \\
2013 & 6 & 28,57 \\
2014 & 4 & 19,04 \\
2015 & 6 & 28,57 \\
2016 & 2 & 9,53 \\
2017 & 1 & 4,76 \\
\hline Total & 21 & 100 \\
\hline
\end{tabular}

Com relação ao delineamento dos artigos selecionados da amostra final, a Tabela 2 apresenta a categorização dos estudos de acordo com os níveis de evidência propostos por Stillwell(10).

Tabela 2 - Delineamento dos estudos segundo nível de evidência

\begin{tabular}{cccc}
\hline Delineamento & Nível de Evidência & $\mathbf{n}$ & $\%$ \\
\hline Revisão Sistemática & I & 0 & 0 \\
Ensaio Controlado Randomizado & II & 5 & 23,8 \\
Ensaio Controlado Não Randomizado & III & 1 & 4,75 \\
Caso Controle ou Estudo de Coorte & IV & 1 & 0 \\
Revisão Sistemática de Estudo Qualitativo ou Descritivo & VI & 14 & 66,7 \\
Estudo Qualitativo ou Descritivo & VII & 0 & 0 \\
Artigo de Opinião ou Consenso de Órgãos Governamentais & & 21 & 100 \\
ou Conselho de Especialidades Médicas & & \\
\hline Total & & 21 \\
\hline
\end{tabular}

\section{Categorização temática}

A leitura dos artigos permitiu categorizar os estudos de acordo com as principais temáticas abordadas pelos diferentes artigos da amostra final. Essas categorias refletem a grande prevalência de estudantes universitários que fazem o uso álcool, as consequências desse uso e a sua utilização como um agente de socialização. Assim, os artigos foram divididos em quatro áreas principais, mostradas na Figura 4, e aqui ressignificadas pelos autores do estudo como: 
Categoria A - Alta prevalência de universitários que utilizam álcool

Essa temática está presente em $72,72 \%(n=16)$ dos artigos. Esses trabalhos evidenciam a alta prevalência de estudantes universitários que fazem uso do álcool. Alguns trabalhos demonstram que a prevalência é maior em estudantes universitários comparados a não estudantes da mesma idade.

\section{Categoria B - Consequências negativas do uso de álcool}

Essa temática está presente em 90,9\% $(n=20)$ dos artigos. Esses trabalhos demonstram as consequências negativas do uso do álcool entre os estudantes universitários. As principais consequências apontadas foram o aumento da mortalidade por fatores externos, como homicídios e acidentes automobilísticos, comportamentos sexuais de risco, abandono dos estudos e risco de dependência futura.

\section{Categoria C - Álcool como agente de} socialização

Essa foi a temática mais fomentada, presente em $95,45 \%(n=21)$ dos artigos. Esses trabalhos evidenciam que uma das principais causas do uso exacerbado de álcool entre os universitários é a sua utilização como agente de socialização.

\section{Categoria D - Modelos motivacionais de beber}

Essa temática está presente em 31,81\% $(n=7)$ dos artigos. Esses trabalhos expõem os modelos motivacionais de beber que afirmam que os indivíduos bebem para aumentar o efeito positivo e diminuir o efeito negativo. Esses motivos incluem os motivos sociais (Figura 4).

\begin{tabular}{|c|c|c|c|}
\hline \multicolumn{2}{|r|}{ Artigo } & \multirow{2}{*}{$\begin{array}{c}\text { Nível de } \\
\text { Evidência }\end{array}$} & \multirow{2}{*}{$\begin{array}{c}\text { Categoria } \\
\text { A } \\
\text { B } \\
\text { C }\end{array}$} \\
\hline 1 & $\begin{array}{l}\text { Natividade JC et al. Fatores de personalidade como preditores do consumo de } \\
\text { álcool por estudantes universitários. Cad Saúde Pública 2012. 28( } 6 \text { ): 1091- } \\
1100^{(11)} \text {. }\end{array}$ & & \\
\hline 2 & $\begin{array}{l}\text { Voogt CV, et al. The Effectiveness of the 'What Do You Drink' Web-based Brief } \\
\text { Alcohol Intervention in Reducing Heavy Drinking among Students: A Two-arm } \\
\text { Parallel Group Randomized Controlled Trial. Alcohol and Alcoholism 2013. 48(3): } \\
312: 321^{(13)} \text {. }\end{array}$ & II & $\begin{array}{l}\text { A } \\
\text { B } \\
\text { C }\end{array}$ \\
\hline 3 & $\begin{array}{l}\text { Rosa LFA, et al. Representações sociais de bebida alcoólica para homens } \\
\text { universitários. Arq. Bras. Psicol. 2015, 67( } 1 \text { ): 3-19(8). }\end{array}$ & VI & $\begin{array}{l}\text { A } \\
\text { B } \\
\text { C }\end{array}$ \\
\hline 4 & $\begin{array}{l}\text { Dumbili et al. Drinking game participation, gender performance and normalization } \\
\text { of intoxication among Nigerian university students Addictive Behaviors Reports } \\
2017 ; 5: 1-8\end{array}$ & VI & $\begin{array}{l}\text { A } \\
\text { B } \\
\text { C }\end{array}$ \\
\hline 5 & $\begin{array}{l}\text { Biolcati R, et al. "I cannot stand the boredom." Binge drinking expectancies in } \\
\text { adolescence. Addictive Behaviors Reports 2017. 3:70-76(12). }\end{array}$ & VI & $\begin{array}{l}B \\
C\end{array}$ \\
\hline 6 & $\begin{array}{l}\text { Helmer SM, et al. Development and evaluation of the efficacy of a web-based } \\
\text { "social norms"-intervention for the prevention and reduction of substance use in a } \\
\text { cluster-controlled trial conducted at eight German universities. BMC Public Healt. } \\
\text { 2016. } 16: 252^{(2)} \text {. }\end{array}$ & III & $\begin{array}{l}\text { A } \\
\text { B } \\
\text { C }\end{array}$ \\
\hline 7 & $\begin{array}{l}\text { Cadigan JM, et al. A latente profile analysis of drinking motives among heavy } \\
\text { drinking college students. Addictive behaviors. } 2015.51: 100-105^{(1)} \text {. }\end{array}$ & VI & $\begin{array}{l}\text { A } \\
B \\
\text { C } \\
\text { D }\end{array}$ \\
\hline 8 & $\begin{array}{l}\text { Dunne EM, Katz EC. Alcohol Outcome Expectancies and Regrettable Drinking- } \\
\text { Related Social Behaviors. Alcohol and Alcoholism (Oxford, Oxfordshire) } 2015 . \\
50(4): 393-398^{(4)} \text {. }\end{array}$ & VI & $\begin{array}{l}\text { A } \\
\text { B } \\
\text { C }\end{array}$ \\
\hline
\end{tabular}




\begin{tabular}{|c|c|c|c|}
\hline & Artigo & Nível de & Categoria \\
\hline 9 & $\begin{array}{l}\text { Young CM, et al. A Longitudinal Examination of the Associations between Shyness, } \\
\text { Drinking Motives, Alcohol Use, and Alcohol-related Problems. Alcoholism, } \\
\text { clinicaland experimental research. } 2015.39(9): 1749-1755^{(3)} \text {. }\end{array}$ & VI & $\begin{array}{l}\text { A } \\
\text { B } \\
\text { C } \\
\text { D }\end{array}$ \\
\hline 10 & $\begin{array}{l}\text { Howard AL, et al. College Student Affect and Heavy Drinking: Variable Associations } \\
\text { Across Days, Semesters, and People. Psychology of addictive behaviors: journal of } \\
\text { the Society of Psychologists in Addictive Behaviors 2015. 29(2):430-443(15). }\end{array}$ & VI & $\begin{array}{l}\text { A } \\
\text { C } \\
\text { D }\end{array}$ \\
\hline 11 & $\begin{array}{l}\text { Moser K, Pearson MR, Hustad JTP, Borsari B. Drinking games, tailgating, and } \\
\text { pregaming: Precollege predictors of risky college drinking. The American jornal of } \\
\text { drugand alcohol abuse. } 2014.40(5): 367-373^{(5)} \text {. }\end{array}$ & II & $\begin{array}{l}\text { A } \\
\text { B } \\
\text { C }\end{array}$ \\
\hline 12 & $\begin{array}{l}\text { Reid AE, et al. Social Network Influences on Initiation and Maintenance of Reduced } \\
\text { Drinking Among College Students. Journal of consulting and clinical psychology. } \\
\text { 2015. 83(1):36-44(6). }\end{array}$ & II & $\begin{array}{l}\text { A } \\
\text { B } \\
\text { C }\end{array}$ \\
\hline 13 & $\begin{array}{l}\text { O'Hara RE, et al. Drinking-to-Cope Motivation and Negative Mood-Drinking } \\
\text { Contingencies in a Daily Diary Study of College Students. Journal of Studies on } \\
\text { Alcohol and Drugs. } 2014 ; 75(4): 606-614^{(16)} \text {. }\end{array}$ & VI & $\begin{array}{l}\text { C } \\
\text { D }\end{array}$ \\
\hline 14 & $\begin{array}{l}\text { Merrill JE, et al. Drinking Reductions following Alcohol-related Sanctions are } \\
\text { associated with Social Norms among College Students. Psychology of addictive } \\
\text { behaviors: journal of the Society of Psychologists in Addictive Behaviors } 2014 \text {. } \\
\text { 28(2):553-558. }\end{array}$ & II & $\begin{array}{l}\text { B } \\
\text { C }\end{array}$ \\
\hline 15 & $\begin{array}{l}\text { Rinker DV, et al. Social influence on temptation: perceived descriptive norms, } \\
\text { temptation and restraint, and problem drinking among college students. Addictive } \\
\text { behaviors } 2013.38(12): 2918-2923\end{array}$ & VI & $\begin{array}{l}\text { A } \\
B \\
\text { C }\end{array}$ \\
\hline 16 & $\begin{array}{l}\text { Lorant V, Nicaise P, Soto VE, d' Hoore W. Alcohol drinking among college students: } \\
\text { college responsibility for personal troubles. BMC Public Health 2013. 13:615 }{ }^{(7)} \text {. }\end{array}$ & VI & $\begin{array}{l}\text { A } \\
\text { B } \\
\text { C } \\
\text { D }\end{array}$ \\
\hline 17 & $\begin{array}{l}\text { Foster DW, et al. Self-consciousness as a moderator of the effect of social drinking } \\
\text { motives on alcohol use. Addictive behaviors 2013. 38(4):1996-2002 }{ }^{(17)} \text {. }\end{array}$ & VI & $\begin{array}{l}\text { A } \\
\text { B } \\
\text { C } \\
\text { D }\end{array}$ \\
\hline 18 & $\begin{array}{l}\text { Terlecki MA, et al. Brief Motivational Intervention for College Drinking: The } \\
\text { Synergistic Impact of Social Anxiety and Perceived Drinking Norms. Psychology of } \\
\text { addictive behaviors: journal of the Society of Psychologists in Addictive Behaviors } \\
\text { 2012. 26(4):917-923(18). }\end{array}$ & II & $\begin{array}{l}\text { A } \\
\text { B } \\
\text { C }\end{array}$ \\
\hline 19 & $\begin{array}{l}\text { Chen Y, et al. Residential environments, alcohol advertising, and initiation and } \\
\text { continuation of alcohol consumption among adolescents in urban Taiwan: A } \\
\text { prospective multilevel study. SSM - Population Health 2016. 2:249-258 }\end{array}$ & VI & $\begin{array}{l}\text { A } \\
B \\
\text { C }\end{array}$ \\
\hline 20 & $\begin{array}{l}\text { Nadkarni A, et al. The explanatory models and coping strategies for alcohol } \\
\text { use disorders: An exploratory qualitative study from India. AsianJournalof } \\
\text { Psychiatry2013. 6(6):521-227(14). }\end{array}$ & VI & B \\
\hline 21 & $\begin{array}{l}\text { Slavinskienè J, et al. Importance of alcohol-related expectations and emotional } \\
\text { expressivity for prediction of motivation to refuse alcohol in alcohol-dependent } \\
\text { patients. Medicina2014. 50(3):169-174 }\end{array}$ & VI & $\begin{array}{l}\text { B } \\
\text { C } \\
\text { D }\end{array}$ \\
\hline 22 & $\begin{array}{l}\text { Pedersen W, et al. Socialization to binge drinking: A population-based, longitudinal } \\
\text { study with emphasis on parental influences. Drug and Alcohol Dependence } 2013 . \\
133(2): 587-592^{(9)} \text {. }\end{array}$ & IV & $\begin{array}{l}\mathrm{B} \\
\mathrm{C}\end{array}$ \\
\hline
\end{tabular}

Figura 4 - Compilação de artigos da amostra final 


\section{Discussão}

O álcool é a droga mais utilizada pela população jovem brasileira, principalmente do sexo masculino. Isso se deve ao fato de que a experimentação tem início precoce e o álcool é de fácil acesso para indivíduos dessa faixa etária ${ }^{(8)}$. Um levantamento realizado no Brasil demonstrou que 53\% dos brasileiros já fizeram uso de álcool; destes, $24 \%$ fazem uso frequente, e essa frequência de consumo é maior ainda quando a amostra é composta por universitários ${ }^{(11)}$. Pesquisas evidenciam que estudantes tendem a consumir uma quantidade maior de álcool do que adultos jovens na mesma faixa etária que não estão estudando. Como os universitários estão mais sujeitos a esse consumo excessivo de álcool, eles também correm um risco especial para as consequências negativas desse uso(2). Estima-se que 1 em 4 estudantes universitários vão abandonar os estudos ou ter outras consequências negativas devido ao uso excessivo de álcool e que 1800 mortes de estudantes a cada ano podem ser relacionadas a lesões atribuídas a esse uso ${ }^{(4)}$.

Dentre as consequências negativas, estão problemas familiares, profissionais e de saúde, comportamentos sexuais de risco e suicídio(7-9). Além disso, o uso abusivo de álcool é considerado uma das principais causas de morte por fatores externos, estando relacionado ao aumento da mortalidade por homicídios e acidentes automobilísticos ${ }^{(8-9)}$. Ademais, o uso em idades precoces aumenta o risco de dependência futura(12). Devido a isso, torna-se necessária a realização de intervenções adequadas ${ }^{(13)}$.

As causas da utilização do álcool por universitários estão relacionadas com fatores externos e internos. Cada vez mais, as bebidas alcoólicas têm sido utilizadas como uma forma de vencer a timidez, aliviar a tensão e propiciar a aproximação entre as pessoas, sendo, então, utilizadas como um agente importante de socialização(8). O maior consumo de álcool entre os universitários em comparação aos não universitários da mesma idade se deve ao fato de que a transição entre o colégio e a faculdade traz mudanças no ajuste dos adolescentes em seu ambiente social( ${ }^{(7)}$. Ademais, ocorre também a influência da ideia culturalmente disseminada de que a faculdade é um momento de independência, controle parental reduzido e uso de álcool(5). Na universidade, o álcool é utilizado em ambientes sociais específicos caracterizados justamente por essa vida independente ${ }^{(7)}$. Além disso, o contato com a bebida alcoólica na faculdade se torna mais frequente, sendo reforçado pelas diversas formas de relações estabelecidas no ambiente universitário(8)
Esse uso de álcool é comum durante os eventos universitários $^{(11)}$, ocorrendo principalmente com os colegas e sendo influenciado por eles. O uso pessoal de álcool está fortemente associado ao uso de álcool entre pares. Geralmente, os indivíduos se filiam a colegas que têm níveis semelhante de consumo de álcool. Isso reflete que uso pessoal de álcool é moldado pelo comportamento dos amigos. Outro fator que evidencia esse fato é que estudantes que querem diminuir o consumo de álcool e manter essa diminuição por um longo período de tempo, provavelmente, terão que alterar sua rede de amizades ${ }^{(6)}$. Um estudo evidenciou que a pressão dos pares é relatada como um dos principais motivos para o uso sustentado do álcool(14).

Os modelos motivacionais de beber afirmam que os indivíduos se envolvem no uso de álcool para atingir um resultado positivo, motivado por necessidades, ou seja, as pessoas bebem por razões específicas em vários contextos, sobretudo para socializar com as outras pessoas. Um modelo bastante utilizado identifica que os principais motivos para o consumo é aumentar o efeito positivo e diminuir o efeito negativo ${ }^{(1,15-16)}$. Dentre esses motivos de beber, estão o motivo de aprimoramento (para criar ou manter um efeito positivo), motivos sociais (para obter resultados sociais positivos), motivos de conformidade (para evitar a rejeição social) e motivos de enfrentamento (para diminuir efeitos negativos) ${ }^{(17)}$.

A ansiedade social pode ser caracterizada pelo medo de rejeição social e/ou medo das interações sociais. Ela está também relacionada com o uso aumentado de álcool entre os universitários. Estudantes com ansiedade social estão mais vulneráveis ao consumo de álcool e aos problemas relacionados com ele. Indivíduos nessas condições utilizam o álcool para evitar a rejeição social dos colegas e/ou porque acreditam que beber é uma estratégia comum para diminuir a ansiedade nessas situações $^{(18)}$.

Outro fator que evidencia a relação entre as relações sociais e o uso de álcool entre universitários é que pessoas com baixos escores em socialização tendem a utilizar mais álcool tanto em frequência quanto em quantidade. A socialização refere-se ao relacionamento interpessoal direcionado ao outro. Pessoas com alto escores de socialização tendem a ser afetuosas, amáveis, cooperativas, altruístas e agradáveis. Já pessoas com baixos escores de socialização tendem a ser pessoas mais fechadas, retraídas e manipuladoras ${ }^{(11)}$.

\section{Conclusão}

Os resultados confirmam que o álcool age como mediador social entre universitários, sendo prevalente 
entre os jovens com maior dificuldade de interações pessoais, que já bebiam no ensino médio, homens e indivíduos com hereditariedade para o alcoolismo.

Todo esse contexto de abuso do álcool entre universitários encontra respaldo nos comerciais expostos na mídia, incentivo da família e de amigos e nos jogos etílicos que estão frequentes nas festas universitárias.

Infelizmente, o excesso de álcool entre universitários configura-se um problema de saúde e educação, por aumento da morbidade e do abandono escolar, respectivamente. O jovem alcoólatra está mais exposto a acidentes de trânsito e inconveniências comportamentais de alto risco à vida. Além disso, o álcool atua como um dos fatores significativos do baixo rendimento escolar ou trancamento e abandono do meio acadêmico.

Por vista das consequências do alcoolismo nas universidades, é imprescindível o aumento de estudos no âmbito, de intervenções na conscientização de jovens, de estratégias legais que limitem atitudes inconsequentes quando em uso do álcool e de atuações das universidades com amparo psicológico e fiscalização, dentro de seus limites físicos, das práticas e incentivos ao alcoolismo.

\section{Referências}

1. Cadigan JM, Martens MP, Herman KC. Latent profile analysis of drinking motives among heavy drinking college students. Addict Behav. 2016; 51: 100-5.

2. Helmer SM, Muellmann S, Zeeb H, Pischke CR. Development and evaluation of the efficacy of a webbased 'social norms'-intervention for the prevention and reduction of substance use in a cluster-controlled trial conducted at eight German universities. BMC Public Health. 2016; 16: 252.

3. Young CM, DiBiello AM, Traylor KZ, Zvolensky MJ, Neighbors C. A Longitudinal Examination of the Associations between Shyness, Drinking Motives, Alcohol Use, and Alcohol-related Problems. Alcohol Clin Exp Res. 2015; 39(9): 1749-55.

4. Dunne EM, Elizabeth CK. Alcohol Outcome Expectancies and Regrettable Drinking-Related Social Behaviors. Alcohol Alcoholism. 2015; 50(4): 393-8.

5. Moser K, Pearson RM, Hustad JTP, Borsari B. Drinking games, tailgating, and pregaming: Precollege predictors of risky college drinking. Am J Drug Alcohol Abuse. 2014 Sep; 40(5): 367-73.

6. Reid AE, Carey KB, Merril JE, Carey MP. Social Network Influences on Initiation and Maintenance of Reduced Drinking Among College Students. J Consult Clin Psychol. 2015; 83(1): 36-44.
7. Lorant V, Nicaise P, Soto VE, D'Hoore W. Alcohol drinking among college students: college responsibility for personal troubles. BMC Public Health. 2013; 13: 615.

8. Rosa LFA, Nascimento ARA. Representações sociais de bebida alcoólica para homens universitários. Arq Bras Psicol. 2015; 67(1):3-19.

9. Pedersen, Soest T. Socialization to binge drinking: A population-based, longitudinal study with emphasis on parental influences. Drug Alcohol Dependence, 2013; 133(2): 587-92.

10. Stillwell SB, Fineout-Overholt E, Melnyk BM, Williamson KM. Evidence-based practice, step by step: searching for the evidence. Am J Nurs. 2010;110(5):41-7.

11. Natividade JC, Aguirre AR, Bizarro L, Hutz CS. Fatores de personalidade como preditores do consumo de álcool por estudantes universitários. Cad Saúde Pública. [Internet]. 2012 [Acesso 1 ago 2017]; 28(6): 1091-100. Disponível em: http://www.scielo.br/scielo.php?script=sci_ arttext\&pid=S0102-311X2012000600008\&lng=en. http:// dx.doi.org/10.1590/S0102-311X2012000600008.

12. Biolcati R, Passini S, Mancini G. I cannot stand the boredom. Binge drinking expectancies in adolescence. Addict Behav Rep. [Internet].2016 [Cited 2017 Aug 1]; 3:706. Available from: http://www.sciencedirect.com/science/ article/pii/S2352853216300116

13. Voogt CV, Poelen EAP, Kleinjan M, Lemmers LACJ, Engels RCME. The Effectiveness of the 'What Do You Drink' Web-based Brief Alcohol Intervention in Reducing Heavy Drinking among Students: A Two-arm Parallel Group Randomized Controlled Trial. Alcohol Alcoholism. [Internet]. 2013 [cited 2017 Aug 1]; 48(3):312-21. Available from: https://academic.oup.com/alcalc/article-lookup/doi/10.1093/ alcalc/ags 133

14. Nadkarni A, Dabholkar H, McCambridge J, Bhat B, Kumar S, Mohanraj R, et al. The explanatory models and coping strategies for alcohol use disorders: An exploratory qualitative study from India. Asian J Psychiatry. [Internet]. 2013 [cited 2017 Aug 1]; 6(6):521-7. Available from: http://www.sciencedirect.com/science/article/pii/ S1876201813001822

15. Howard AL, Patrick ME, Maggs JL. College Student Affect and Heavy Drinking: Variable Associations Across Days, Semesters, and People. Psychol Addictive Behav. [Internet]. 2015 [cited 2017 Aug 1]; 29(2):430-43. doi:10.1037/adb0000023. Available from: https://www. ncbi.nlm.nih.gov/pmc/articles/PMC4411211/

16. O'Hara RE, Armeli S, Tennen H. Drinking-to-Cope Motivation and Negative Mood-Drinking Contingencies in a Daily Diary Study of College Students. J Studies Alcohol Drugs. [Internet]. 2014 [cited 2017 Aug 1]; 75(4):606-14. Available from: https://www.ncbi.nlm.nih.gov/pmc/articles/ PMC4108601/ 
17. Foster DW, Neighbors C. Self-consciousness as a moderator of the effect of social drinking motives on alcohol use. Addictiv Behav. [Internet]. 2013 [cited 2017 Aug 1]; 38(4):1996-2002. Available from: https://www.ncbi.nlm.nih. gov/pmc/articles/PMC4215938/

18. Terlecki MA, Buckner JD, Larimer ME, Copeland AL. Brief Motivational Intervention for College Drinking: The Synergistic Impact of Social Anxiety and Perceived Drinking Norms. Psychol Addict Behav. [Internet] 2012 [cited 2017

Aug 1]; 26(4):917-23. Available from: https://www.ncbi.nlm. nih.gov/pmc/articles/PMC3427392/ 